\title{
Predictive Value of Serum Myoglobin and Creatine Phosphokinase for Development of Acute Kidney Injury in Traumatic Rhabdomyolysis
}

\author{
Nithin Abraham Raju, Shoma Vinay Rao, J. Chakravarthy Joel', Gijoe George Jacob, Arun Kunnanchery Anil, S. Mahasampath Gowri², \\ Subramani Kandasamy \\ Division of Critical Care, Christian Medical College, Vellore, Tamil Nadu, 'Department of Anaesthesia, Bangalore Baptist Hospital, Bengaluru, Karnataka, ${ }^{2}$ Department of \\ Biostatistics, Christian Medical College, Vellore, Tamil Nadu, India
}

\section{Abstract}

Introduction: Rhabdomyolysis (RM) is a condition where there is injury to striated muscle fibers causing release of myoglobin, creatine phosphokinase (CPK), and other intracellular contents into the circulation. High myoglobin levels cause acute kidney injury (AKI). Trauma is the most common cause of RM and development of complications related to the degree of myoglobin released. Currently, the degree of $\mathrm{RM}$ is assessed and treatment is instituted based on serum CPK. As myoglobin is the direct cause of AKI, we set out to determine if serum myoglobin is a more reliable predictor than CPK for the development of AKI in traumatic RM. Methodology: A prospective observational study of 90 patients was admitted to the surgical Intensive Care Unit/high dependency unit of a tertiary hospital with traumatic RM whose serum CPK $>5000 \mathrm{U} / \mathrm{L}$. Along with standard treatment including intravascular volume optimization and hemodynamic stabilization, they were treated with "crush protocol." Daily/twice a day, serum CPK and myoglobin were estimated. Categorical data are expressed as frequency and percentage, and the continuous variables are presented as mean (standard deviation) or median (interquartile range) based on normality. Other statistical analyses were done using the Chi-square test, independent $t$-test, and rank sum test based on normality. Results: Fourteen out of 90 patients developed AKI and one patient required renal replacement therapy. CPK value of $>12,000 \mathrm{U} / 1$ was identified to have $64 \%$ sensitivity and $56 \%$ specificity for developing AKI whereas serum myoglobin value of $>5000 \mathrm{ng} / \mathrm{ml}$ was identified to have $78 \%$ sensitivity and $77 \%$ specificity for developing AKI. Conclusion: Following traumatic RM, in patients on "crush protocol," serum myoglobin is a more sensitive and specific test than serum CPK, for predicting AKI.

Keywords: Acute kidney injury, crush protocol, myoglobin, rhabdomyolysis

\section{INTRODUCTION}

Rhabdomyolysis (RM) is a clinical syndrome secondary to skeletal muscle injury. ${ }^{[1-4]}$ The products of muscle breakdown, notably, myoglobin cause renal failure. ${ }^{[1-4]}$ Traditionally, serum creatine phosphokinase (CPK) is being measured to assess and manage RM to prevent acute kidney injury (AKI). ${ }^{[4]}$ When myoglobin is the direct cause of renal injury, estimation of serum myoglobin than $\mathrm{CPK}$, a surrogate marker in RM, would be more reliable in assessing the risk of AKI severity and guide the management of RM. ${ }^{[5]}$ This study is an attempt to establish serum myoglobin levels which would predict establishment of renal failure in RM, compared to serum CPK in patients treated with "crush protocol."

\begin{tabular}{|l|l|}
\hline \multicolumn{3}{c|}{ Access this article online } \\
\hline Quick Response Code: & Website: \\
& www.ijccm.org \\
\cline { 2 - 2 } & \\
\end{tabular}

\section{Aims}

The primary aim of the study is to determine if serum myoglobin (being implicated as the direct cause of AKI in traumatic RM) is a more reliable marker than CPK for the development of AKI in traumatic RM. Secondary aims are:

a. To determine serum myoglobin level which could predict development of AKI

Address for correspondence: Dr. Shoma Vinay Rao, Division of Critical Care, Christian Medical College, Vellore - 632 004, Tamil Nadu, India. E-mail: shovinrao@gmail.com

This is an open access article distributed under the terms of the Creative Commons Attribution-NonCommercial-ShareAlike 3.0 License, which allows others to remix, tweak, and build upon the work non-commercially, as long as the author is credited and the new creations are licensed under the identical terms.

For reprints contact: reprints@medknow.com

How to cite this article: Raju NA, Rao SV, Joel JC, Jacob GG, Anil AK, Gowri SM, et al. Predictive value of serum myoglobin and creatine phosphokinase for development of acute kidney injury in traumatic rhabdomyolysis. Indian J Crit Care Med 2017;21:852-6. 
b. To determine need for renal replacement therapy (RRT) in this subset of patients who develop AKI

c. To determine duration of mechanical ventilation (MV), Intensive Care Unit length of stay (ICULOS), and inhospital mortality in patients with and without AKI secondary to RM.

\section{Methodology}

Permission from the Institutional Review board and ethics committee approval were obtained and the study was registered with the clinical trials registry - India (CTRI/2012/08/002892). A prospective observational study, including 90 patients with traumatic RM, admitted to the surgical ICU/high dependency unit of a tertiary referral hospital was conducted. Inclusion criteria: age (18-70 years) and trauma-induced RM (excluding compartment syndrome). Exclusion criteria: patients with chronic renal failure and RM due to other etiologies.

Informed consent was obtained from the awake nonventilated patients, or an assent was obtained from relatives in sedated ventilated patients. All patients with diagnosis of trauma were screened, and they were recruited when the serum CPK level was $>5000 \mathrm{IU} / \mathrm{L}$ during screening or subsequently during the course of ICU admission. All recruited patients received standard treatment including intravascular volume optimization and hemodynamic stabilization along with initiation of "crush protocol" [Figure 1] when the serum CPK $>5000 \mathrm{IU} / \mathrm{L}$. Serum myoglobin (Roche diagnostics electrochemiluminescence assay) was estimated along with CPK. Urine $\mathrm{pH}$ was determined $8^{\text {th }}$ hourly as part of crush protocol. Renal function was monitored with hourly urine output and serum creatinine on everyday basis. AKI was diagnosed using AKI Network (AKIN) criteria. ${ }^{[6]}$ Blood gas analysis, serum sodium, and potassium were done at least once a day. Serum magnesium and phosphate were estimated based on the clinical situation. Decision on RRT was made based on discussion with nephrologists.

The discriminating capacity to determine AKI using peak serum myoglobin and peak CPK levels was analyzed using the ROC curve, and optimal cutoff values were chosen. Categorical data were expressed as frequency and percentages. Continuous variables are presented as mean (standard deviation) or median (interquartile range) based on normality. The relationship between categorical variables and AKI was analyzed using Chi-square test. The relationship between continuous variables and AKI was analyzed using the independent $t$-test or rank sum test based on normality. All data were analyzed using STATA I/C 13.1 version (Stata Corp LLC, Texas, USA).

\section{RESULTS}

A total of 90 patients, including $75(83.33 \%)$ males and $15(16.67 \%)$ females, aged between 15 and 69 years (mean $34.21 \pm 13.64)$, were recruited. $14 / 90(15.55 \%)$ patients developed AKI. There were more men than women. In patients with traumatic RM, the mean APACHE II score at ICU admission was $14.31 \pm 5.96$ for patients who did not develop AKI versus $17.83 \pm 8.70$ for patients who developed AKI. The difference was not statistically significant $(P=0.3916)$.

Mean peak CPK level in patients who did not develop AKI patients was $16650.89 \pm 19237.54 \mathrm{U} / \mathrm{L}$ versus $29170.07 \pm 31383.75 \mathrm{U} / \mathrm{L}$ in patients who developed AKI, demonstrating a statistically significant difference $(P=0.0317)$. Of the 14 patients who developed AKI, ten had Stage 1 and two each Stage 2 and Stage 3 renal failure. The mean peak serum myoglobin values among patients who developed AKI and who did not develop AKI were $5112.86 \pm 8794.74 \mathrm{ng} / \mathrm{mL}$ and $23104.79 \pm 43709.20 \mathrm{ng} / \mathrm{mL}$, respectively $(P=0.0007)$. This was statistically significant at $1 \%$ level.

To predict AKI in traumatic RM patients, peak serum myoglobin value of $\geq 5160 \mathrm{ng} / \mathrm{ml}$ was found to have $78.57 \%$ sensitivity and $79.17 \%$ specificity, and peak CPK value of $\geq 12,388 \mathrm{U} / \mathrm{L}$ was found to have $64.29 \%$ sensitivity and $59.21 \%$ specificity [Graph 1]. One patient who developed AKI needed RRT.

The mean number of days of MV was $3.70 \pm 3.59$ and $6.18 \pm 6.69$ among non-AKI and AKI patients, respectively $(P=0.3030)$ [Table 1]. Mean ICU LOS was $5.41 \pm 3.24$ days

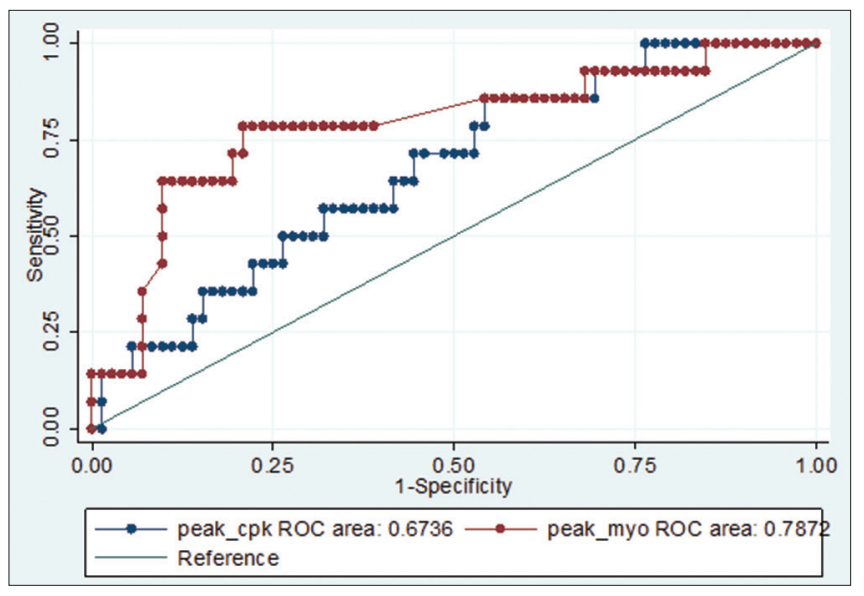

Graph 1: Receiver operator characteristic curve creatine phosphokinase/ myoglobin

\begin{tabular}{|c|c|c|c|}
\hline & Non-AKI & AKI & $P$ \\
\hline Number of patients & 76 & 14 & \\
\hline APACHE II & $14.31 \pm 5.96$ & $17.83 \pm 8.70$ & 0.3916 \\
\hline $\begin{array}{l}\text { Duration of MV } \\
\text { (days) }\end{array}$ & $3.70 \pm 3.59$ & $6.18 \pm 6.69$ & 0.3030 \\
\hline ICU LOS (days) & $5.41 \pm 3.24$ & $12.09 \pm 18.66$ & 0.4498 \\
\hline Peak CPK (U/L) & $16,650.89 \pm 19,237.54$ & $29,170.07 \pm 31,383.75$ & 0.0317 \\
\hline $\begin{array}{l}\text { Peak myoglobin } \\
(\mathrm{ng} / \mathrm{ml})\end{array}$ & $5112.86 \pm 8794.74$ & $23,104.79 \pm 43,709.20$ & 0.0007 \\
\hline Mortality & 23 & 5 & 0.686 \\
\hline
\end{tabular}

APACHE II: Acute Physiology and Chronic Health Evaluation II; MV: Mechanical ventilation; ICU: Intensive Care Unit; LOS: Length of stay; CPK: Creatine phosphokinase; AKI: Acute kidney injury 


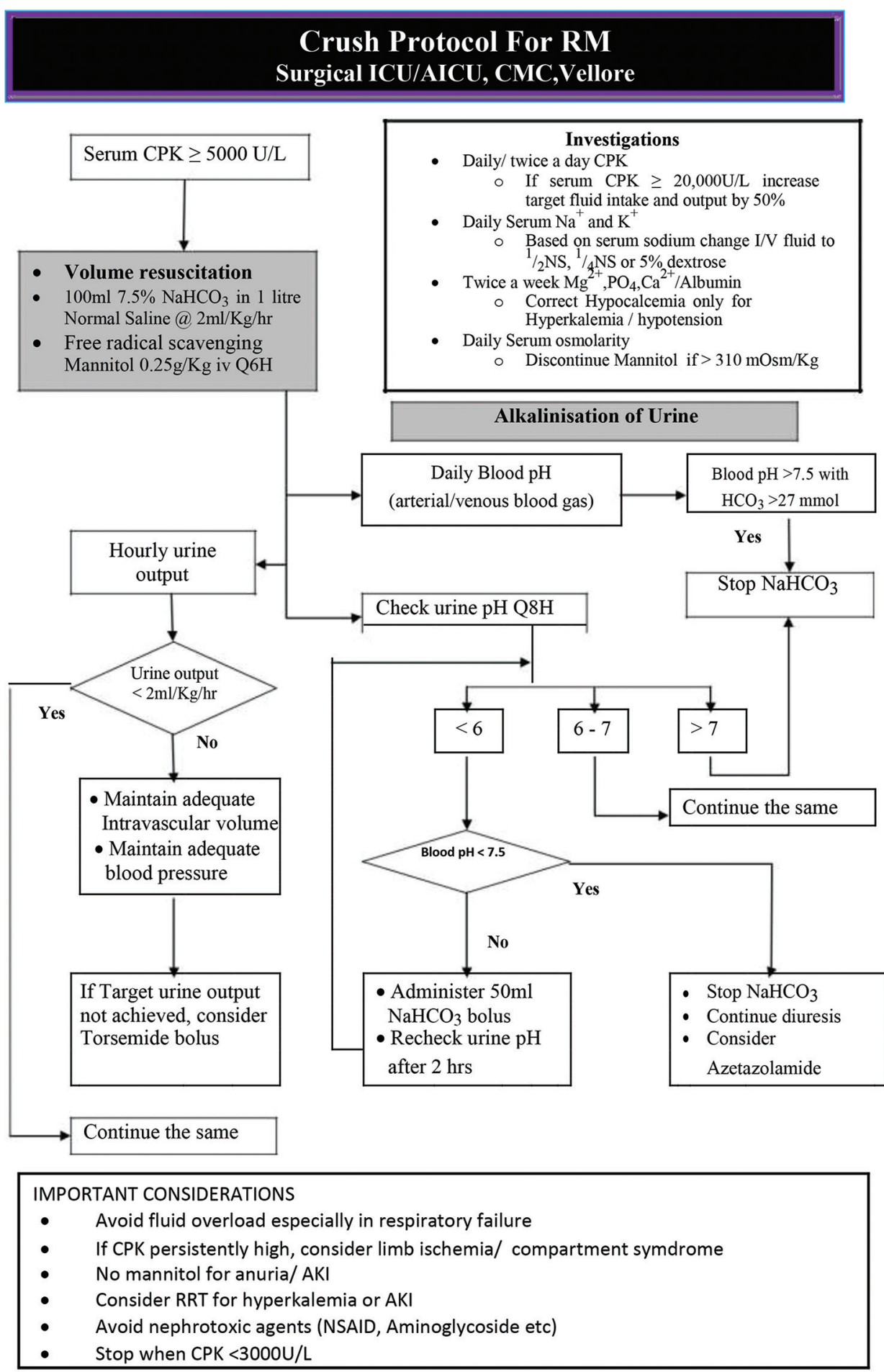

Figure 1: Crush Protocol

in the non-AKI group against $12.09 \pm 18.66$ in the AKI group $(P=0.4498)$ [Table 1].

The overall mortality of patients with traumatic RM was $(28 / 90) 31 \%$. In patients who did not develop AKI, the mortality was (23/76) $30.26 \%$ versus (5/14) $35.7 \%$ in patients who developed AKI [Table 1]. This was not statistically significant $(P=0.686)$.

\section{DISCUSSION}

RM is a clinical syndrome characterized by injury to skeletal muscle fibers with disruption and release of their contents, namely, myoglobin, CPK, and lactate dehydrogenase into the circulation..$^{[1]}$ Myoglobin, which is similar to hemoglobin, is the most toxic of these, and release of massive amounts can result in glomerular obstruction and AKI. ${ }^{[2]}$ Other 
postulated mechanisms are renal vasoconstriction, lipid peroxidation, and damage due to oxygen-free radicals. RM has been identified as a leading cause of AKI (13\%-50\% cases of RM develop AKI), but AKI caused by RM has a relatively good prognosis (mortality rates for AKI due to RM approximate $20 \%$ ). ${ }^{[1-3]}$ Traumatic RM or crush syndrome is the most common cause of RM (approximately $85 \%$ of major trauma victims develop RM) followed by strenuous exercise, extremes of temperature, drugs/toxins, electrical or other burns, infection, vascular injuries, prolonged immobilization malignant hyperthermia, and electrolyte abnormalities. ${ }^{[1,3]}$ The severity of RM relates to the degree of myoglobin released and can cause complications such as disseminated intravascular coagulopathy, compartment syndrome, and sequestration of fluid from the circulation into edematous muscles leading to hypovolemia and shock. ${ }^{[1]}$ Metabolic derangements such as hyperkalemia, metabolic acidosis, hypocalcemia or hypercalcemia, hyperuricemia, hyponatremia, hypomagnesemia, and hyperphosphatemia with increased risk of cardiac arrhythmias are not uncommon. ${ }^{[4]}$

Diagnosis of RM is based on the presence of myoglobinuria (dark urine) and elevated serum CPK levels. In large case series, ${ }^{[3,5]}$ mean peak CPK levels were 10,000-25,000 U/L in all causes except malignant hyperthermia, where values approximated 60,000 U/L. Many authors have identified CPK levels $>5$ times of normal has been shown to be diagnostic of $\mathrm{RM}$ and levels $>5,000 \mathrm{U} / \mathrm{L}$ has been shown to have a strong correlation for development of AKI. ${ }^{[3,6-8]}$ The half-life of serum CPK is 36 hours, the levels rise 2 to 12 hours of injury, peaking at 24 to 72 hours. The levels typically reduce to $50 \%$ by 24 hours with adequate treatment ${ }^{[3,6,7]}$ and arrest of RM. Other causes of elevated CPK include muscular dystrophies, nerve damage, heart disease, thyroid disorders, and renal insufficiency ${ }^{[9]}$

Historically, increased serum myoglobin and myoglobinuria have been used for the diagnosis of RM albeit poor sensitivity and specificity. ${ }^{[6]}$ Normal serum level of myoglobin is $<85 \mathrm{ng} / \mathrm{mL} \cdot{ }^{[7,8]}$ Myoglobin is rapidly metabolized in the liver and excreted by the kidneys. With a half life of 2 to 3 hours, serum myoglobin returns to normal by 6 to 8 hours. This makes myoglobin an early marker of RM and also to monitor treatment. ${ }^{[3,6,7,10]}$ Serum myoglobin levels also increase in myocardial infarction and levels correlate with infarct size. ${ }^{[1]]}$ Myoglobin being a heavier molecule $(17,800 \mathrm{Da})$ is cleared better with ultrafiltration than dialysis. ${ }^{[5,10]}$ High flux dialysis may be employed to clear myoglobin than conventional dialysis. Diagnosis of renal failure has undergone major changes in the last two decades. There are at least three new criteria described, namely, Risk, Injury, Failure, Loss and End Stahe (RIFLE), Acute Kidney Injury Network (AKIN) and Kidney Disease: Improving global outcome (KDIGO) for the diagnosis and Classification of AKI.

Renal failure in critically ill patients can be multifactorial. In trauma, hypovolemic shock is a leading cause of renal failure. RIFLE, AKIN, and KDIGO systems of classification are used currently for diagnosis and classification of renal failure. We chose AKIN system as it does not have creatinine clearance and uses the baseline creatinine as reference value, making it easier to use at bedside. KDIGO scoring came into existence after we commenced our study. All our patients were hemodynamically optimized with fluids and vasoactive medications as quickly as feasible. Among 14 patients who developed AKI, 10 (71.4\%) patients had Stage 1, 2 patients each (14.3\%) Stage 2 and 3 AKI. Of the two who developed Stage 3 AKI, one needed RRT.

Our center uses "crush protocol" for management of patients with RM [Figure 1]. ${ }^{[6,12]}$ Albeit myoglobin is implicated as the main cause of AKI in RM, crush protocol uses the surrogate marker CPK (>5000 IU/L) for initiation and maintenance of therapy for RM. Serum CPK levels remain high for a longer period of time than serum myoglobin levels, making CPK a better marker for diagnosis of muscle damage than serum myoglobin. ${ }^{[10]}$

Crush protocol involves infusion of large volumes of intravenous fluids to induce diuresis. This could lead to fluid overload, generalized edema, and respiratory failure. It also includes use of diuretics and alkalinizing agents which can lead to metabolic and electrolyte derangements. Fluid overload could affect the respiratory system in terms of mechanics with reduced compliance and reduced oxygenation. In our study, there was no difference in duration of MV in patients who developed AKI compared to who did not develop AKI. The judicious use of the crush protocol might have led to this outcome. There was no difference in the duration of MV, ICU length of stay, and mortality in patients between the two groups.

Data analysis revealed statistically significant higher peak serum CPK and myoglobin values among patients who developed AKI. Different authors have mentioned various cutoff values of both serum CPK and myoglobin for the development of AKI, but none of them mention treatment with crush protocol [Figure 1]. ${ }^{[13]}$ In a retrospective series of multifactorial RM, Premru et al., ${ }^{[14]}$ myoglobin values $>15 \mathrm{mg} / \mathrm{L}(15,000 \mathrm{ng} / \mathrm{ml})$ were significantly associated with the development of renal failure and need for hemodialysis. In our series, only one patient needed dialysis and therefore could not be tested statistically. In patients identified to have traumatic RM, who receive treatment with crush protocol [Figure 1], serum myoglobin value is more sensitive and specific for predicting the development of AKI. The incidence of AKI due to traumatic RM (15\%) is similar to those observed by previous authors. ${ }^{[2,7,8]}$ Treatment with crush protocol [Figure 1] has probably reduced the incidence and progress of AKI due to traumatic RM in our patients. Peak serum myoglobin values $\geq 5000 \mathrm{ng} / \mathrm{ml}$ have $78 \%$ sensitivity and $79 \%$ specificity for the development of AKI whereas peak CPK values $\geq 12,000 \mathrm{U} / \mathrm{L}$ have only $64 \%$ sensitivity and $59 \%$ specificity. This is in favor of our hypothesis that serum myoglobin is a better predictor of AKI than CPK.

\section{Conclusion}

Following traumatic RM, in patients on crush protocol, serum myoglobin is a more sensitive and specific test than serum CPK 
for predicting AKI. Myoglobin level $>5000 \mathrm{ng} / \mathrm{L}$ is associated with an increased risk of AKI. In traumatic RM, estimation of serum myoglobin is useful to monitor the response to therapy and estimate the risk of AKI and thus useful to prognosticate AKI.

\section{Financial support and sponsorship}

Financial support was provided by the IRB Christian Medical College, Vellore - 632 004, Tamil Nadu, India.

\section{Conflicts of interest}

There are no conflicts of interest.

\section{References}

1. Petejova N, Martinek A. Acute kidney injury due to rhabdomyolysis and renal replacement therapy: A critical review. Crit Care 2014;18:224.

2. Vanholder R, Sever MS, Erek E, Lameire N. Rhabdomyolysis. J Am Soc Nephrol 2000;11:1553-61.

3. Huerta-Alardín AL, Varon J, Marik PE. Bench-to-bedside review: Rhabdomyolysis - An overview for clinicians. Crit Care 2005;9:158-69.

4. Zimmerman JL, Shen MC. Rhabdomyolysis. Chest 2013;144:1058-65.

5. Melli G, Chaudhry V, Cornblath DR. Rhabdomyolysis: An evaluation of
475 hospitalized patients. Medicine (Baltimore) 2005;84:377-85.

6. Khan FY. Rhabdomyolysis: A review of the literature. Neth J Med 2009;67:272-83.

7. Mikkelsen TS, Toft P. Prognostic value, kinetics and effect of CVVHDF on serum of the myoglobin and creatine kinase in critically ill patients with rhabdomyolysis. Acta Anaesthesiol Scand 2005;49:859-64.

8. Beetham R. Biochemical investigation of suspected rhabdomyolysis. Ann Clin Biochem 2000;37(Pt 5):581-7.

9. Octura JE, Lee KJ, Cho HW, Vega RS, Choi J, Park JW, et al. Elevation of blood creatine kinase and selected blood parameters after exercise in thoroughbred racehorses (Equus caballus L.). J Res Agric Anim Sci 2014:2:7-13.

10. Zhang MH. Rhabdomyolosis and its pathogenesis. World J Emerg Med 2012;3:11-5.

11. Stone MJ, Waterman MR, Harimoto D, Murray G, Willson N, Platt MR, et al. Serum myoglobin level as diagnostic test in patients with acute myocardial infarction. Br Heart J 1977;39:375-80.

12. Sever MS, Vanholder R, Lameire N. Management of crush-related injuries after disasters. N Engl J Med 2006;354:1052-63.

13. Kuzmanovska B, Cvetkovska E, Kuzmanovski I, Jankulovski N, Shosholcheva M, Kartalov A, et al. Rhabdomyolysis in critically ill surgical patients. Med Arch 2016;70:308-10.

14. Premru V, Kovač J, Ponikvar R. Use of myoglobin as a marker and predictor in myoglobinuric acute kidney injury. Ther Apher Dial 2013;17:391-5. 